\title{
Backbone and side chain NMR assignments of the H-NOX domain from Nostoc sp. in complex with BAY58-2667 (cinaciguat)
}

\author{
Garyfallia I. Makrynitsa ${ }^{1}$ - Aikaterini I. Argyriou ${ }^{1} \cdot$ Georgios Dalkas ${ }^{1} \cdot$ Dimitra A. Georgopoulou ${ }^{1} \cdot$ Marina Bantzi $^{2,3}$. \\ Athanassios Giannis ${ }^{2} \cdot$ Andreas Papapetropoulos $^{4} \cdot$ Georgios A. Spyroulias $^{1}$ (D)
}

\begin{abstract}
Soluble guanylate cyclase (sGC) enzyme is activated by the gaseous signaling agent nitric oxide (NO) and triggers the conver-sion of GTP (guanosine 5'-triphosphate) to cGMP (cyclic guanylyl monophosphate). It contains the heme binding H-NOX (heme-nitric oxide/oxygen binding) domain which serves as the sensor of NO and it is highly conserved across eukaryotes and bacteria as well. Many research studies focus on the synthesis of chemical compounds bearing possible therapeutic action, which mimic the heme moiety and activate the sGC enzyme. In this study, we report a preliminary solution NMR (Nuclear Magnetic Resonance) study of the H-NOX domain from Nostoc sp. cyanobacterium in complex with the chemical sGC activator cinaciguat (BAY58-2667). An almost complete sequence-specific assignment of its ${ }^{1} \mathrm{H}$, ${ }^{15} \mathrm{~N}$ and ${ }^{13} \mathrm{C}$ resonances was obtained and its secondary structure predicted by TALOS + .
\end{abstract}

Keywords H-NOX domain · Soluble guanylate cyclase (sGC) · NMR spectroscopy · Cinaciguat · BAY58-2667

\section{Biological context}

Soluble guanylate cyclase (sGC) is considered as a very attractive therapeutic target for cardiovascular diseases due to its implication to the NO/cGMP signaling pathway (Erdmann et al. 2013). Nitric oxide (NO) acts as a key regulatory molecule of the sGC enzyme, upon binding on the N-terminal H-NOX (heme-nitric oxide/oxygen binding) domain. This event leads to the activation of sGC and subsequently to the conversion of GTP (guanosine 5'-triphosphate) to cGMP (cyclic guanylyl monophosphate) (Ma et al.

Garyfallia I. Makrynitsa and Aikaterini I. Argyriou have equally contributed to this work.

$\triangle$ Georgios A. Spyroulias

G.A.Spyroulias@upatras.gr

1 Department of Pharmacy, University of Patras, 26504 Patras, Greece

2 Institut für Organische Chemie, Universität Leipzig, Johannisallee 29, 04103 Leipzig, Germany

3 Department of Chemistry, University of Fribourg, Chemin du Musée 9, 1700 Fribourg, Switzerland

4 Laboratory of Pharmacology, Faculty of Pharmacy, National and Kapodistrian University of Athens, Athens, Greece
2007; Underbakke et al. 2014). Loss of the heme cofactor of H-NOX domain and low levels of NO bioavailability are responsible for the malfunction of the NO/cGMP pathway resulting to heart disease, neurodegeneration, hypertension and erectile dysfunction as well (Breitenstein et al. 2016; Friebe et al. 2007; Gheorghiade et al. 2013).

Two classes of chemical compounds have characterized to act towards SGC in a NO-independent manner, stimulators and activators. Stimulators are heme-dependent compounds while activators act on a heme free or heme-oxidized sGC (Follmann et al. 2013; Nossaman et al. 2012). Stimulators like Riociguat bind upon the reduced heme sGC potentiating the action of NO when the bioavailability is low. Although stimulators can activate the enzyme in absence of NO, they act also synergistically with the gas (Evgenov et al. 2006; Grimminger et al. 2009). On the other hand, activators like cinaciguat (BAY58-2667) occupies the same cavity as the heme of H-NOX domain after its oxidation resulting in a fully activated enzyme (Erdmann et al. 2013; Evgenov et al. 2006).

Our research group has published previously the backbone and the side chain NMR (Nuclear Magnetic Resonance) resonance assignments of the H-NOX domain from Nostoc. $s p$ cyanobacterium (Ns H-NOX) (Alexandropoulos et al. 2016) revealing that $N s \mathrm{H}-\mathrm{NOX}$ is a well-folded protein 
in solution with a stable structure. Herein, we report the backbone and side chain NMR resonance assignments of the Ns H-NOX domain in complex with the activator cinaciguat (BAY58-2667).

\section{Methods and experiments}

\section{Protein sample preparation}

The experimental conditions for $N s$ H-NOX (residues 1-183) expression and purification have been stated in the previous study (Alexandropoulos et al. 2016). Heme replacement by cinaciguat has been performed as described elsewhere (Martin et al. 2010).

\section{NMR spectroscopy}

The solvent system of all the protein samples for NMR measurements contains $90 \% \mathrm{H}_{2} \mathrm{O}$ and $10 \% \mathrm{D}_{2} \mathrm{O}$. The protein for both samples $\left({ }^{15} \mathrm{~N}\right.$ and ${ }^{15} \mathrm{~N} /{ }^{13} \mathrm{C}$ ) was $1 \mathrm{mM}$ and all NMR experiments acquired on a Bruker Avance III HD four channel $700 \mathrm{MHz}$ NMR spectrometer at 298K, equipped with TCI $5 \mathrm{~mm}{ }^{1} \mathrm{H} /{ }^{13} \mathrm{C} /{ }^{15} \mathrm{~N} / \mathrm{D}$-gradient probe.

The sequence specific assignments of the Ns H-NOX/ cinaciguat complex were obtained by applying common backbone and side assignment methods using the following heteronuclear NMR experiments: 2D ${ }^{1} \mathrm{H}_{-}{ }^{15} \mathrm{~N}$ HSQC (heteronuclear single-quantum coherence) $\&{ }^{1} \mathrm{H}_{-}{ }^{13} \mathrm{C} \mathrm{HSQC}$ and 3D HNCO, 3D HN(CA)CO, 3D TROSY-HN(CO)CACB, 3D TROSY-HNCACB, 3D (H)CCH-TOCSY aliphatic, 3D HNHA, 3D ${ }^{15} \mathrm{~N}$-NOESY, 3D ${ }^{13} \mathrm{C}-\mathrm{NOESY}$ aliphatic \& aromatic (Wüthrich 1986). The NMR data were processed with Topspin 3.2 pl5 software and analyzed with CARA (Keller 2004).

\section{Extent of assignments and data deposition}

The ${ }^{1} \mathrm{H}_{-}{ }^{15} \mathrm{~N}$ HSQC spectrum of the Ns H-NOX/cinaciguat shows that the complex forms a properly folded protein with a stable structure in solution (Fig. 1a-d). A complete set of NMR experiments performed using standard parameters for biomolecular NMR, described in the previous section, led to the identification and to the sequential assignment of $96 \%$ of the protein backbone nuclei, $73.5 \%$ of all the nuclei and $60.1 \%$ of the side chain nuclei. Specifically, it has been assigned $98.3 \%$ of all $\mathrm{C}_{\alpha}$ nuclei, $97.6 \%$ of all $\mathrm{C}_{\beta}$ nuclei, $98.3 \%$ of all $\mathrm{CO}$ nuclei, $85 \%$ of all $\mathrm{H}_{\beta}$ and $92.4 \%$ of all $\mathrm{H}_{\alpha}$ nuclei. However, no backbone amide resonances could be detected for residues M1, L4, V38, G39, D45, H124, S126, S127. All the chemical shifts of the complex have been deposited in the BioMagResBank (http://www.bmrb.wisc.edu) under the accession no. 28118. These assignment percentages' are significantly higher than the corresponding ones for the Ns H-NOX in native (heme-bound) state ( $82 \%$ backbone nuclei and $64.5 \%$ of all nuclei) (Alexandropoulos et al. 2016) implying that the cinaciguat complex forms a more stable structure in solution.

The identification of the cis/trans conformation of the proline residues was based on the analysis of the 3D TROSY HNCACB and 3D (H)CCH-TOCSY aliphatic. The values of the chemical shift differences $\Delta \beta \gamma=\delta\left[13 C_{\beta}\right]-\delta\left[13 C_{\gamma}\right]$ (Schubert et al. 2002) for each proline revealed that P62, P95, P113, P118 and P143 exist in trans conformation. Due to the limited information for P117, its conformation could not be determined.

Chemical shifts of $\mathrm{Ns} \mathrm{H}-\mathrm{NOX} /$ cinaciguat were introduced to TALOS+ (Shen et al. 2009) server for the secondary structure prediction. According to TALOS + prediction (Fig. 2b), it adopts a structure composed by $7 \alpha$-helices and $4 \beta$-strands arranged in $\alpha \alpha \alpha \alpha \alpha \alpha \beta \beta \alpha \beta \beta$ topology, identical to those observed in the crystal structure of the Ns H-NOX/ cinaciguat complex (PDB ID: 3L6J, Fig. 2a) (Martin et al. 2010). Based on the CATH server (Dawson et al. 2017), the complex falls into the alpha-beta complex architecture category, a quite typical fold type for H-NOX domains (Makrynitsa et al. 2019). However, slight differences are detected, comparing to the TALOS + results of native $N s$ H-NOX ( $5 \alpha$-helices and $5 \beta$-strands) (Fig. 2c) (Alexandropoulos et al. 2016). These differences concern mainly the helices above and below the heme moiety due to the missing residues in the native $N s \mathrm{H}-\mathrm{NOX}$.

Overall, this contribution presents a solution NMR study of the Nostoc sp. H-NOX domain in complex with the sGC activator, cinaciguat (BAY58-2667), by applying molecular biology protocols and 3D triple resonance NMR experiments. The ${ }^{1} \mathrm{H}_{-}{ }^{15} \mathrm{~N}$ HSQC spectrum is characterized by signal dispersion while the complete sequence-specific assignment of the protein resonances revealed a well-folded domain with mixed $\alpha / \beta$ secondary structure elements, which allows the identification of more resonances (73.5\%) under the same experimental condition, compared to the native, heme-bound, Nostoc sp. The predicted secondary structure of the cinaciguat complex is in full agreement with the available crystal structure and comprises $4 \beta$-strands and 7 $\alpha$-helices while it is quite close to the corresponding one of the heme-bound Ns H-NOX. Further NMR studies of the Ns H-NOX/cinaciguat will allow us to investigate its solution structure and dynamics. Such data may contribute to the biochemical and functional characterization of H-NOX domains. 


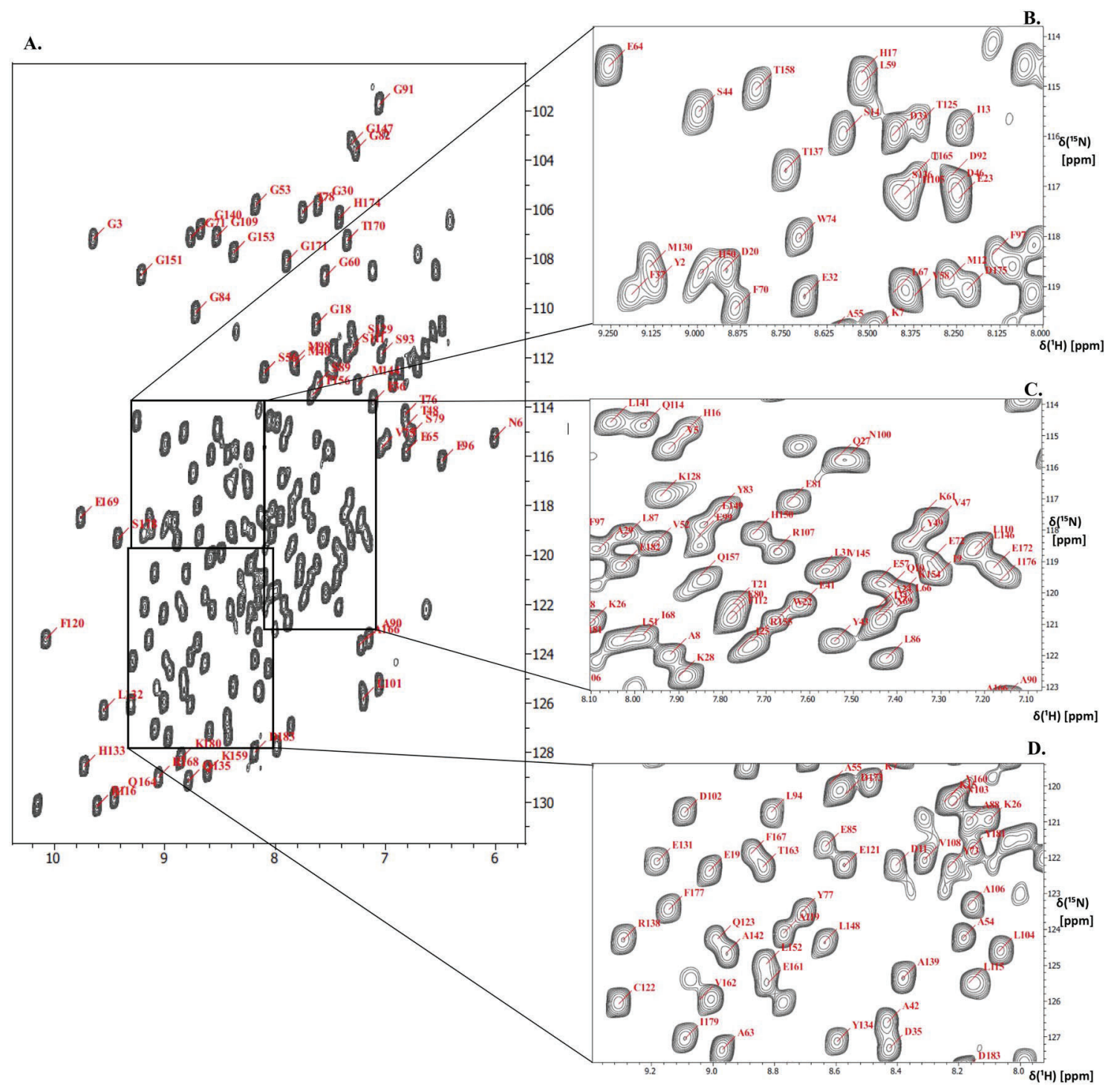

Fig. 1 a $700 \mathrm{MHz} 2 \mathrm{D}{ }^{1} \mathrm{H}^{-15} \mathrm{~N}$ HSQC spectrum at $298 \mathrm{~K}$ of uniformly labeled ${ }^{15} \mathrm{~N}$-labeled Ns H-NOX/cinaciguat complex. Resonance assignments are numbered according to the sequence of Nostoc sp.

protein. b-d Magnification of the central regions of the HSQC spectrum as indicated 


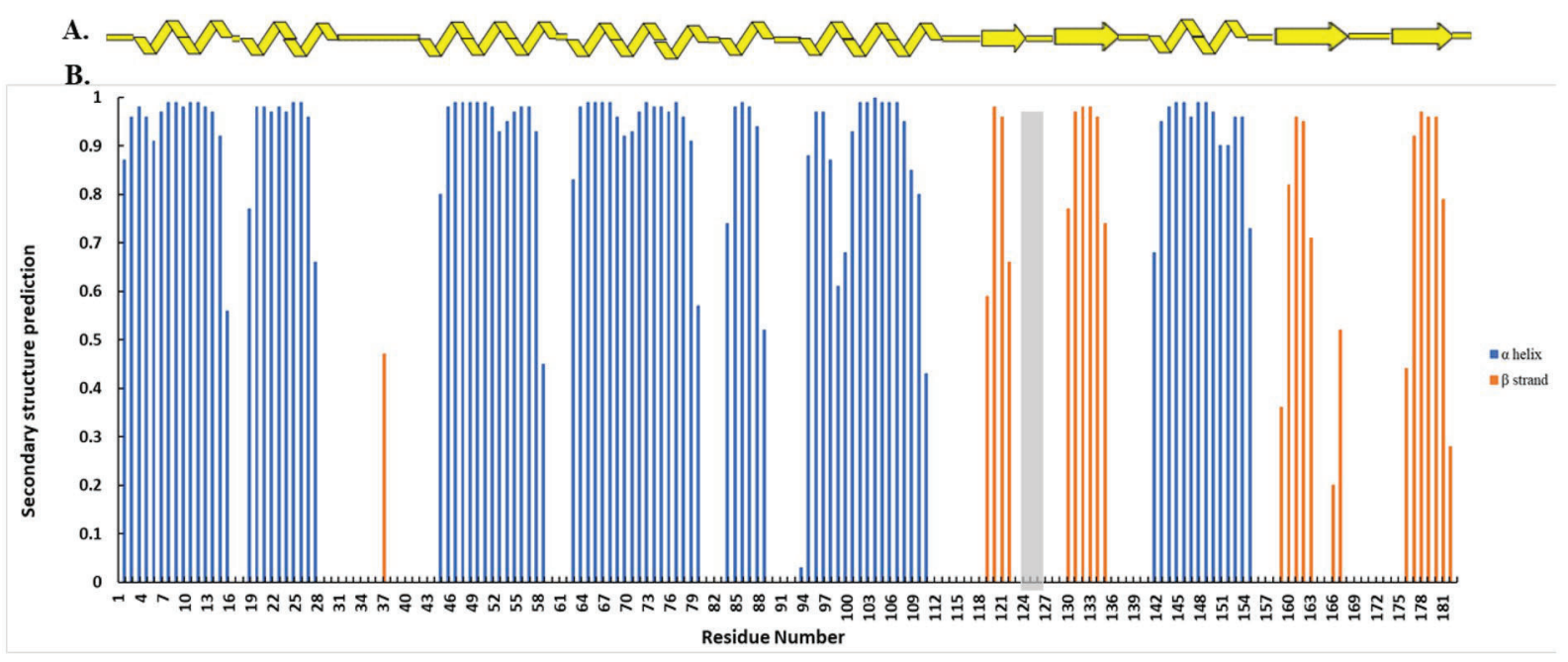

C.

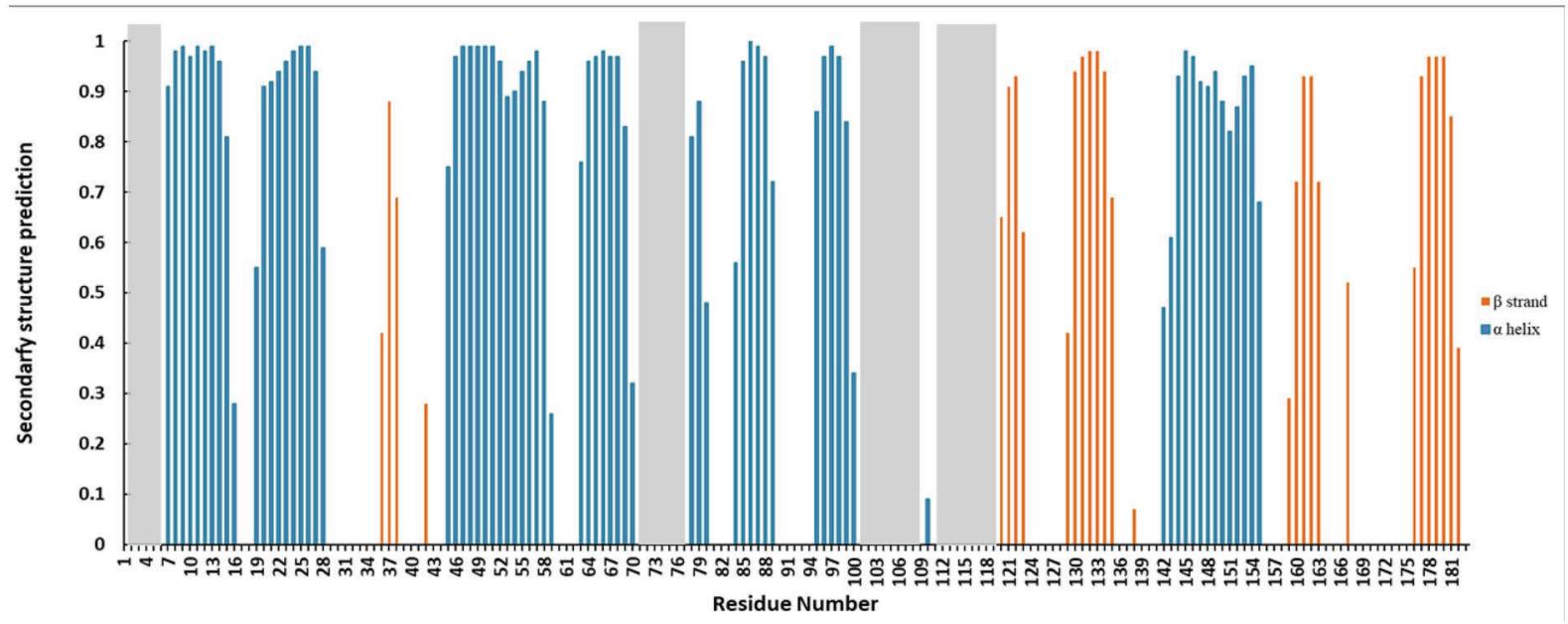

Fig. 2 a Secondary structure of $N s$ H-NOX/cinaciguat complex as determined from the crystal structure (PDB ID: 3L6J). b Secondary structure of $N s \mathrm{H}-\mathrm{NOX} /$ cinaciguat complex as predicted by TALOS+ from the chemical shift values (blue and orange bars indicate $\alpha$-helix and $\beta$-strand, respectively). c Secondary structure of the heme-bound
Ns H-NOX as predicted by TALOS+ from the chemical shift values (blue and orange bars indicate $\alpha$-helix and $\beta$-strand, respectively) (Alexandropoulos et al. 2016). Grey bars indicate the unassigned residues
Author contribution Garyfallia I. Makrynitsa and Aikaterini I. Argyriou have equally contributed to this work.

Data availability Assignment deposited in BMRB with ID: 28,118.

\section{Compliance with ethical standards}

Conflict of interest The authors declare no competing financial interest.

\section{References}

Alexandropoulos II, Argyriou AI, Marousis KD, Topouzis S, Papapetropoulos A, Spyroulias GA (2016) 1H, 13C, 15N backbone and side-chain resonance assignment of Nostoc sp. C139A variant of the heme-nitric oxide/oxygen binding (H-NOX) domain. Biomol NMR Assign 10:395-400

Breitenstein S, Roessig L, Sandner P, Lewis KS (2016) Novel sGC stimulators and $\mathrm{sGC}$ activators for the treatment of heart failure. Heart failure. Springer, Cham, pp 225-247 
Dawson NL et al (2017) CATH: an expanded resource to predict protein function through structure and sequence. Nucleic Acids Res 45:D289-D295

Erdmann E, Semigran MJ, Nieminen MS, Gheorghiade M, Agrawal R, Mitrovic V, Mebazaa A (2013) Cinaciguat, a soluble guanylate cyclase activator, unloads the heart but also causes hypotension in acute decompensated heart failure. Eur Heart J 34:57-67

Evgenov OV, Pacher P, Schmidt PM, Haskó G, Schmidt HH, Stasch J-P (2006) NO-independent stimulators and activators of soluble guanylate cyclase: discovery and therapeutic potential. Nat Rev Drug Discov 5:755-768

Follmann M et al (2013) The chemistry and biology of soluble guanylate cyclase stimulators and activators Angewandte. Chem Int Ed 52:9442-9462

Friebe A, Mergia E, Dangel O, Lange A, Koesling D (2007) Fatal gastrointestinal obstruction and hypertension in mice lacking nitric oxide-sensitive guanylyl cyclase. Proc Nat Acad Sci 104:7699-7704

Gheorghiade M et al (2013) Soluble guanylate cyclase: a potential therapeutic target for heart failure. Heart Fail Rev 18:123-134

Grimminger F et al (2009) First acute haemodynamic study of soluble guanylate cyclase stimulator riociguat in pulmonary hypertension. Eur Respir J 33:785-792

Keller RL (2004) CANTINA VERLAG. The computer aided resonance assignment tutorial. 1st edn

Ma X, Sayed N, Beuve A, Van Den Akker F (2007) NO and CO differentially activate soluble guanylyl cyclase via a heme pivotbend mechanism. EMBO J 26:578-588
Makrynitsa GI, Zompra AA, Argyriou AI, Spyroulias GA, Topouzis S (2019) Therapeutic targeting of the soluble guanylate cyclase. Curr Med Chem 26:2730-2747

Martin F et al (2010) Structure of cinaciguat (BAY 58-2667) bound to Nostoc H-NOX domain reveals insights into hememimetic activation of the soluble guanylyl cyclase. J Biol Chem 285:22651-22657

Nossaman B, Pankey E, Kadowitz P (2012) Stimulators and activators of soluble guanylate cyclase: review and potential therapeutic indications. Crit Care Res Pract. https://doi. org/10.1155/2012/290805

Schubert M, Labudde D, Oschkinat H, Schmieder P (2002) A software tool for the prediction of Xaa-Pro peptide bond conformations in proteins based on $13 \mathrm{C}$ chemical shift statistics. J Biomol NMR 24:149-154

Shen Y, Delaglio F, Cornilescu G, Bax A (2009) TALOS+: a hybrid method for predicting protein backbone torsion angles from NMR chemical shifts. J Biomol NMR 44:213-223

Underbakke ES, Iavarone AT, Chalmers MJ, Pascal BD, Novick S, Griffin PR, Marletta MA (2014) Nitric oxide-induced conformational changes in soluble guanylate cyclase. Structure 22:602-611

Wüthrich K (1986) NMR with proteins and nucleic acids. Europhys News 17:11-13 